\title{
Article
}

\section{Atomistic Explanation of the Dramatically Improved Oxygen Reduction Reaction of Jagged Platinum Nanowires, 50 times better than Pt}

\author{
Yalu Chen, Tao Cheng, and William A. Goddard
}

J. Am. Chem. Soc., Just Accepted Manuscript • DOI: 10.1021/jacs.9b13218 • Publication Date (Web): 22 Apr 2020

Downloaded from pubs.acs.org on April 22, 2020

\section{Just Accepted}

"Just Accepted" manuscripts have been peer-reviewed and accepted for publication. They are posted online prior to technical editing, formatting for publication and author proofing. The American Chemical Society provides "Just Accepted" as a service to the research community to expedite the dissemination of scientific material as soon as possible after acceptance. "Just Accepted" manuscripts appear in full in PDF format accompanied by an HTML abstract. "Just Accepted" manuscripts have been fully peer reviewed, but should not be considered the official version of record. They are citable by the Digital Object Identifier (DOIB). "Just Accepted" is an optional service offered to authors. Therefore, the "Just Accepted" Web site may not include all articles that will be published in the journal. After a manuscript is technically edited and formatted, it will be removed from the "Just Accepted" Web site and published as an ASAP article. Note that technical editing may introduce minor changes to the manuscript text and/or graphics which could affect content, and all legal disclaimers and ethical guidelines that apply to the journal pertain. ACS cannot be held responsible for errors or consequences arising from the use of information contained in these "Just Accepted" manuscripts. 


\section{INTRODUCTION}

Proton Exchange Membrane Fuel Cells (PEMFCs) provide the most promising means for addressing the global renewable energy supply and clean environment ${ }^{1-3}$. Currently the main impediment for large-scale PEMFCs commercialization is the sluggish oxygen reduction reactions (ORR), which dramatically increases catalyst costs. Extensive efforts are underway to develop electrocatalysts for ORR with much higher performance and lower $\cos ^{4-6}$. Pt-alloy nanomaterials show enhanced performance ${ }^{7-12}$, especially $\mathrm{PtNi}$ alloys ${ }^{13-17}$. In particular, very dramatically improved performance has been observed in dealloyed Pt nanomaterials ${ }^{18-22}$. Thus Debe and coworkers showed that $\mathrm{Ni}_{7} \mathrm{Pt}_{3}$ nanoparticles (NP) led to significantly improved ORR activity, 4 times higher than pure $\mathrm{Pt}$ and $60 \%$ higher than for the NSTF standard $\mathrm{Pt}_{68} \mathrm{Co}_{29} \mathrm{Mn}_{3}$ alloy ${ }^{23-26}$. However, no Ni was observed in the XPS, suggesting the NP was a dealloyed Pt NP. Fortunelli and Goddard (FG) used the ReaxFF reactive force field ${ }^{27}$ to predict the structure of the dealloyed Debe NP and found that starting with $70 \% \mathrm{Ni}$, led to a porous $\mathrm{Pt}$ with significant interior area exposed to the surface. $\mathrm{FG}$ found that the $\mathrm{O}_{\text {ads }}$ hydration reaction,

$\mathrm{O}_{\text {ads }}+\mathrm{H}_{2} \mathrm{O}_{\mathrm{ads}} \rightarrow 2 \mathrm{OH}_{\text {ads }}$ (1)

which is the rate determining step (RDS) on Pt (111), is dramatically accelerated on this porous NP compared to Pt $(111)^{28}$.

Later, Huang, Duan, Goddard, and co-workers ${ }^{29}$ showed that dealloying $5 \mathrm{~nm} \mathrm{Ni}_{7} \mathrm{Pt}_{3}$ Nanowires (NW) led to $2 \mathrm{~nm}$ Pt Jagged NW (J-PtNW) with 50 times faster ORR than current state-of- the-art $\mathrm{Pt} / \mathrm{C}$, but they found that all Ni was extracted! Building this dramatically improved performance into commercial fuel cells would be a game-changer, dramatically decreasing the costs of hydrogen fuel cells needed to extract the power from fuel generated and stored at solar power plants to provide practical transportation without $\mathrm{CO} 2$ emissions.

Although several factors, such as under-coordinated surface $\mathrm{Pt}$ atoms, mechanical strain, and high electrochemically active surface area (ECSA), have been suggested to contribute to this remarkably accelerated ORR activity ${ }^{29}$, there was no atomistic explanation about which sites on this very complex surface are responsible, leaving no roadmap to build this activity into practical electrodes.

To address this conundrum, we report here multiscale simulations to explain the dramatically improved performance and to characterize the atomic features of active sites responsible. Starting with the J-PtNW synthesized computationally using ReaxFF reactive force field, we selected randomly 500 of the more than 10,000 surface sites and carried out Quantum Mechanics (QM) Calculations on clusters within $8 \AA$ of the surface site. Here we considered reaction (1), the RDS on Pt (111). We observed a strong correlation between OO Distance (the distance between $\mathrm{O}_{\text {ads }}$ and the $\mathrm{O}$ of $\mathrm{H}_{2} \mathrm{O}_{\text {ads }}$ ) and the free energy barrier for reaction (1). Indeed $14.4 \%$ of the 500 surface sites sampled are barrier-less of reaction (1) at room temperature, compared to $0.29 \mathrm{eV}$ for $\mathrm{Pt}$ (111) and hence increased reaction rate. We then modeled the reaction rates of all surface sites based on their OO Distances and estimated the performance of the whole J-PtNW. 
We found two distinct geometric patterns

- Triangles where the $\mathrm{H} 2 \mathrm{O}$ binds to one vertex and $\mathrm{O}$ to the other two;

- Concave-Up Rhombi where $\mathrm{O}$ binds to one edge and the $\mathrm{H} 2 \mathrm{O}$ to one of the remaining two vertices.

The atomic structure of these active sites provides insights on designing high-performance electrocatalysts for ORR.

\section{RESULTS AND DISCUSSION}

\subsection{Structure Analysis and Surface Extraction of J-PtNW}

Starting with the J-PtNW synthesized using ReaxFF reactive molecular dynamics (RMD) as in the previous work $^{29}$, we identified all surface atoms using the surface vector based methodology. The detailed description of structure synthesis and surface vector based methodology are listed in S1 and S2 of Supporting Information. The nanowire has $6926 \mathrm{Pt}$ atoms, with 3881 on the surface (surface ratio: $56.0 \%$ ). We partitioned these surface sites into coordination groups based on the number of first-neighbor atoms, using a first-neighbor cut-off at $3.50 \AA$ based on the radial distribution functions in Figure S2(a) of Supporting Information. The J-PtNW showed broader peaks with the first peak located close to the first peak of Pt single crystal, as expected. We observed many undercoordinated and over-coordinated sites both on the surface and in the bulk. The surface site distribution versus coordination is shown in Figure S2(b) of Supporting Information. For better visualization, we plotted the surface in Figure S3 of Supporting Information, with all surface sites colored by their coordination (ranging from 3 to 12). This structural analysis showed that the J-PtNW have an extremely disordered and irregular surface, making it challenging to characterize experimentally and computationally.

\subsection{Bridge Nanocluster Model}

To study the relationship between the structure and catalytic activity, we need to define a physical descriptor that might correlate with the performance (reaction rate). Based on the reaction mechanism revealed by earlier work ${ }^{30-32}$, we focused on the $\mathrm{O}_{\text {ads }}$ hydration, reaction (1).

Our full solvent QM Metadynamics showed that this step is the rate determining step (RDS) for applied potentials of $U \leq 0.9 \mathrm{~V}$ reversible hydrogen electrode (RHE) $)^{30,33,34}$. Since this reaction requires two sites for water adsorption and oxygen adsorption, instead of the single site nanocluster model used in our previous machine learning studies for Copper ${ }^{35,36}$ and Gold ${ }^{37,38}$, we developed a new bridge nanocluster model in this work. As shown in Figure 1, we generated the bridge nanocluster by cutting two spheres of size $\mathrm{R}$ around the $\mathrm{Pt}$ atom for water adsorption (Pt-1) and the Pt atom for oxygen adsorption (Pt-2). Then, we merged these two spheres to a single nanocluster. The choice of $\mathrm{R}$ was based on the benchmark calculations in $\mathbf{S 3}$ of Supporting Information. We found that $8.0 \AA$ is already sufficient to provide $0.02 \mathrm{eV}$ accuracy in the adsorption energy for both reactants and products. Therefore, we consider that 8.0 $\AA$ provides the best balance between accuracy and efficiency and used this cut-off throughout this work.

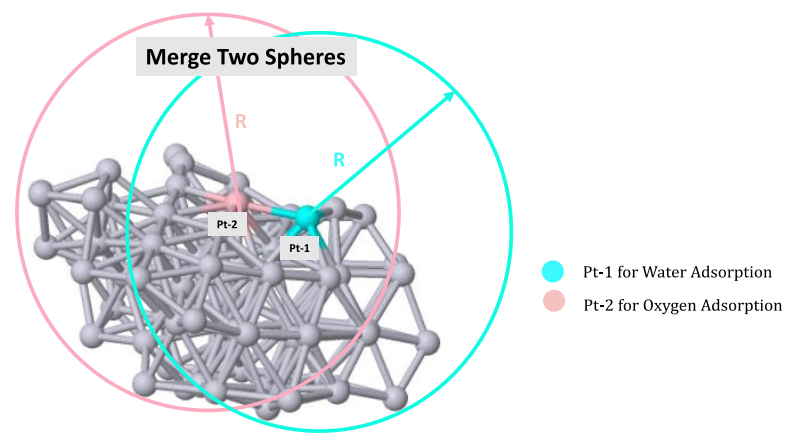

Figure 1. Bridge Nanocluster Model. We first cut two spheres of size R around Pt-1 (cyan atom) and Pt-2 (pink tom), where Pt-1 is specifically for water adsorption and $\mathrm{Pt}-2$ is for oxygen adsorption. These two spheres are then merged to a single nanocluster. $\mathrm{R}$ here is optimized to $8 \AA$. This bridge nanocluster will later be used for DFT calculations.

\subsection{OO Distance as Physical Descriptor}

We carried out DFT calculations ${ }^{39}$ for the $\mathrm{O}_{\text {ads }}$ hydration, reaction (1), using the bridge nanocluster model defined in Section 2.2. We chose the free energy barrier $\left(G_{a}\right)$ of reaction (1) as the physical descriptor to evaluate the performance of each bridge nanocluster. We expect that sites with lower $G_{a}$ should have better ORR performance. With such a disordered and irregular surface, we might have to sample all bridging surface sites to be sure to properly represent the activity of the full NW. DFT calculations on the transition state reaction barrier for the whole J-PtNW, would require far too much computational resources ${ }^{40}$. Instead we defined a physical descriptor, OO Distance (d-OO), the distance between the O of $\mathrm{H}_{2} \mathrm{O}_{\text {ads }}$ and the $\mathrm{O}_{\text {ads. }}$. Then we showed that d-OO is highly correlated with the free energy barrier $\left(G_{a}\right), \mathrm{G}_{\mathrm{a}}=\mathrm{G}_{\mathrm{TS}}-$ $\mathrm{G}_{\text {Reactants, but much faster to calculate. }}$

To obtain d-OO, we need only to optimize the structure of the reactants, H2O and O. We show in Figure S7 of Supporting Information three examples illustrating the correlation between d-OO and $G_{a}$ at room temperature. By comparing to our reference case $\mathrm{Pt}$ (111), which has $G_{a}=0.29 \mathrm{eV}$ and d-OO $=3.36 \AA$, we observed that shorter d-OO leads to much lower $G_{a}$ (d-OO at $2.60 \AA$ leads to $G_{a}=0.00 \mathrm{eV}$ ) and larger d-OO leads to higher $G_{a}$ (d-OO at $3.98 \AA$ leads to $G_{a}=0.35 \mathrm{eV}$ ). In addition, thermodynamic reaction energy $\left(\Delta \mathrm{G}=\mathrm{G}_{\text {Products }}-\mathrm{G}_{\text {Reactants }}\right)$, doesn't correlate with $G_{a}$, as expected. Therefore, we used d$\mathrm{OO}$ as the physical descriptor to evaluate performance of each bridge nanocluster. All our calculations include solvation effect using the VASPsol implicit solvation model ${ }^{41}$. As shown in Figure S5 and S6 in Supporting Information, solvation affects $\mathrm{d}-\mathrm{OO}$ in a non-negligible manner. The free energy barriers $\left(G_{a}\right)$ at room temperature $(298.15 \mathrm{~K})$ are obtained by adding to the DFT electronic energy (E), the zero-point energy (ZPE), enthalpy and entropy contribution from vibrational modes of surface species. More computational details of d-OO and $G_{a}$ are listed in $\mathbf{S 4}$ and $\mathbf{S 5}$ of Supporting Information.

\subsection{Data Sampling for DFT calculations (d-OO)}

To generate all possible bridge pairs from 3881 surface $P$ t atoms of the J-PtNW, we first examined Pt-1 for water adsorption of all surface Pt atoms. Then, for each Pt-1, we examined its first- 
neighbors on the surface to select Pt-2 for binding an $\mathrm{O}$ atom. This defines a bridge pair. The definition of Pt-1 and Pt-2 are the same as in Figure 1. Then, we cut the corresponding bridge nanoclusters from the NW. This generates the same nanocluster twice with Pt-1 and Pt-2 swapping the labels, but we consider them as different structures since Pt-1 is specifically for water adsorption and $\mathrm{Pt}-2$ is specifically for oxygen adsorption. The two Pts are not identical when binding adsorbates. An example of two nanoclusters of same coordinates but defined as different structures is shown in Figure S8 of Supporting Information.

In this way, we generated 21,057 bridge pairs. Since $\mathrm{H} 2 \mathrm{O}$ binds weakly to high-coordination sites, we expect that they would be inactive for ORR. To test this, we selected randomly 50 sites for which Pt-1 has a coordination larger than 9 and calculated water adsorption. We found water desorbed from all 50 sites. Therefore, we discarded the high-coordination bridge pairs (coordination of Pt-1 > 9), which leaves 12,400 bridge nanoclusters for further sampling. This procedure is Illustrated schematically in Figure 2.

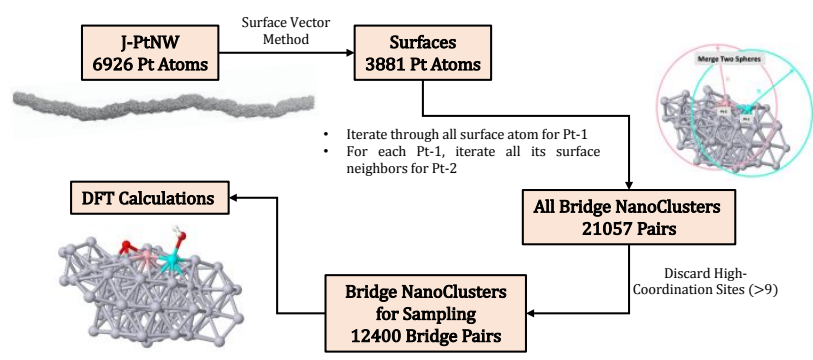

Figure 2. Generating All Bridge Pairs for Data Sampling. Starting from the J-PtNW with $6926 \mathrm{Pt}$ atoms, we identified 3881 surface atoms using surface vector methodology. Then, we iterated first over the whole surface for Pt-1 and for each Pt-1 we iterated over its surface neighbors for Pt-2. In this way, we generated a total number of 21,057 bridge pairs. We showed that sites with coordination larger than 9 , cannot adsorb water. Discarding these bridge pairs with coordination of Pt-1 larger than 9 leaves 12,400 bridge pairs for data sampling.

We then randomly sampled 500 bridge nanoclusters from these 12,400 pairs for DFT calculations. For each nanocluster, we put water on Pt-1 and oxygen on Pt-2, and then optimized the structure to find the best conformation for the adsorbates. Solvation effect are included here using the VASPsol implicit solvation model. Additional DFT calculation details, including all benchmark calculations are listed in Section S4, Table S1 S3 and Figure S5 S6 of Supporting Information. The sampled bridge centers are plotted back on the NW in Figure S9 of Supporting Information. The bridge centers are distributed throughout the whole nanowire, indicating our sample is a good representation of the whole J-PtNW.

The distribution of d-OO among 500 nanoclusters is shown in Figure 3. We see that d-OO ranges from $2.50 \AA$ to $6.01 \AA$. However, $35.2 \%$ of the sites have d-OO distance shorter than the d-OO (3.36 $\AA$ ) for Pt (111). We expect these sites to have much lower free energy barrier for $\mathrm{O}_{\text {ads }}$ hydration and hence to contribute significantly to the dramatically improved performance of J-PtNW at ORR. To help provide a better understanding of how the geometry of different sites affects d$\mathrm{OO}$ and free energy barrier, several representative structures with different d-OO are also shown at the bottom of Figure 3.
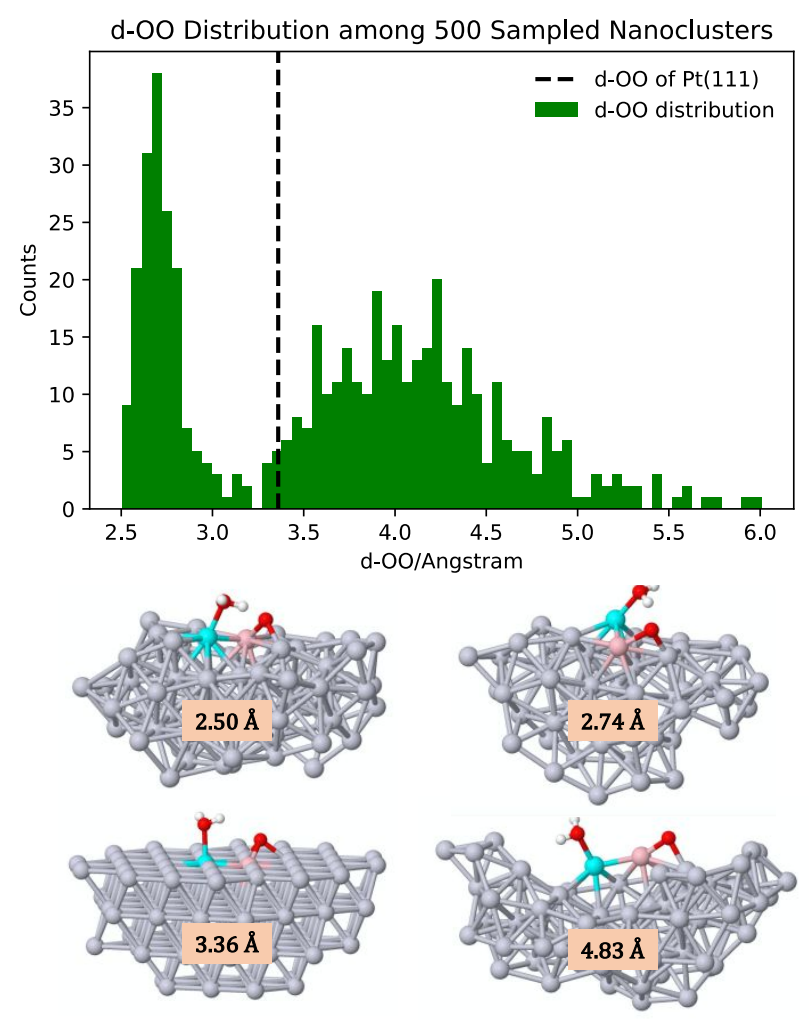

Figure 3. d-OO Distribution among 500 Sampled Bridge Nanoclusters. Our sample showed a broad range of d-OO, from $2.50 \AA$ to $6.01 \AA$. The d-OO of Pt (111) is marked in the plot as the dashed line. We observed $35.2 \%$ of the sites lying to the left to $\mathrm{Pt}$ (111), showing much shorter d-OO. We expect these sites to have much lower energy barrier for $O_{a d s}$ hydration, thus leading to higher ORR performance. Four representative structures with different d-OO are attached here for understanding the correlation between structure and catalytic activities.

\subsection{Correlation of d-OO with Catalytic Activity}

To further understand the correlation between d-OO and the catalytic activity of each bridge site, we selected 18 structures with various d-OO and calculated the transition state and free energy barriers. The structures were selected randomly and independently but in such a way to ensure that we covered a broad range of d-OO. For each structure, we carried out the transition state search using the climbing image nudged elastic band (NEB) method with implicit solvation using VASPsol tool. The free energy barriers $\left(G_{a}\right)$ for the $O_{\text {ads }}$ hydration at room temperature $(298.15 \mathrm{~K})$ are then obtained by adding to the DFT electronic energy (E), the zero-point energy (ZPE), enthalpy and entropy contribution from vibrational modes of surface species. The data for the 18 structures, including d-OO and free energy barriers $\left(G_{a}\right)$ at room temperature are listed in Table 1. More computation details toward the transition state search are included in S5 of Supporting Information. 
Table 1. Correlation Between d-OO and Free Energy Barrier $\left(G_{a}\right)$ at Room temperature 298.15K

\begin{tabular}{cc|cc|cc}
$\mathrm{d}-\mathrm{OO} / \AA$ & $\mathrm{G}_{\mathrm{a}}(298.15 K, \mathrm{eV})$ & $\mathrm{d}-\mathrm{OO} / \AA$ & $\mathrm{G}_{\mathrm{a}}(298.15 K, \mathrm{eV})$ & $\begin{array}{c}\mathrm{d}-\mathrm{OO} / \AA \\
3.50\end{array}$ & $\mathrm{G}_{\mathrm{a}}(298.15 K, \mathrm{eV})$ \\
2.50 & 0.00 & 2.76 & 0.01 & 3.57 & 0.20 \\
2.56 & 0.00 & 2.80 & 0.06 & 3.70 & 0.19 \\
2.60 & 0.00 & 2.93 & 0.12 & 3.75 & 0.14 \\
2.64 & 0.00 & 2.99 & 0.10 & 3.98 & 0.40 \\
2.68 & 0.00 & 3.12 & 0.16 & & 0.35 \\
2.74 & 0.01 & $\underline{3.36}$ & $\underline{0.29}$ & & 0.28
\end{tabular}

We observed a strong correlation between d-OO and $G_{a}$, as shown in Table 1. The sites with small d-OO shows low free energy barriers, especially with d-OO $<=2.68 \AA, O_{a d s}$ hydration step becomes barrier-less. And with larger d-OO, $G_{a}$ generally becomes higher, but in a non-linear manner. Pt (111) shows d-OO at $3.36 \AA$ and $G_{\mathrm{a}}$ at $0.29 \mathrm{eV}$, underlined in Table 1. To better correlate d-OO with catalytic activity, we used transition state theory (TST) to estimate the reaction rate as $k=$ $\left(k_{B} T / h\right) \exp \left(-\mathrm{G}_{\mathrm{a}} / \mathrm{k}_{\mathrm{B}} \mathrm{T}\right)$. The reaction rates are plotted versus d-OO of 18 structures in Figure 4. Here, we used the sigmoid function to fit the $\mathrm{k}(\mathrm{d}-\mathrm{OO})$ curve at room temperature. The fitted curve is also shown in Figure 4 with $R^{2}$ at 0.9894 .

Therefore, we found that a large portion of sites on the J-PtNW surface exhibit dramatically improved performance toward $\mathrm{O}_{\mathrm{ads}}$ hydration, the RDS. We claim that this is the main contributor to the dramatically improved ORR performance. In addition, we showed that $\mathrm{d}-\mathrm{OO}$ is a good descriptor for evaluating catalytic activity of any specific bridge pair. Appendix of the Supporting Information provides coordinates of the pathway for three representative structures from Table 1.

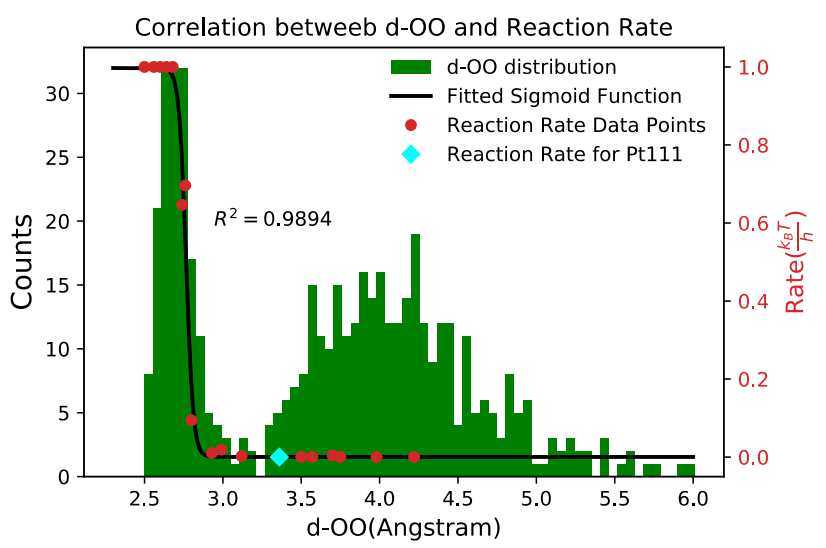

Figure 4. Correlation Between d-OO and Reaction Rate. we used transition state theory (TST) to estimate the reaction rate as $k=$ $\left(k_{B} T / h\right) \exp \left(-G_{a} / k_{B} T\right)$ of 18 selected structures listed in Table 1. The reaction rates are plotted in the unit of $\left(k_{B} T / h\right)$ versus d-OO. We then used the sigmoid function to fit the $\mathrm{k}(\mathrm{d}-\mathrm{OO})$ curve at room temperature, $k=L /\left(1+\exp \left(A \times\left(d-O O-x_{0}\right)\right)\right)+b$. The parameters at $\left(A=47.1056, x_{0}=2.7659, b=0.0003, L=0.9968\right)$ gives the $R^{2}=0.9894$.

\subsection{Performance Prediction of the Whole J-PtNW}

Since our dataset is sampled randomly and independently, we consider it to be representative of the whole J-PtNW surface. Thus, we estimated the performance of the whole nanowire by mapping the statistics of our sample back to the full NW. The
J-PtNW has $6926 \mathrm{Pt}$ atoms with 3881 surface atoms, which leads to 21,057 bridge pairs. Among these 21,057 pairs, 8657 are high-coordination involved pairs, which we assume are inactive. The remaining 12,400 pairs should have the similar dOO distribution as the 500 samples.

To estimate the performance improvement at different temperatures, we first calculated the free energy barriers at different temperature, and refit the sigmoid functions. In other words, $\mathrm{k}(\mathrm{d}-\mathrm{OO})$ is dependent of T. Three examples of fitting $\mathrm{k}(\mathrm{d}-\mathrm{OO})$ at different temperatures are shown in Figure S10 of Supporting Information.

$k(d-O O \mid T)=L /\left(1+\exp \left(A \times\left(d-O O-x_{0}\right)\right)\right)+b(2)$

Then, the total performance of J-PtNW is calculated by mapping the sample statistic to total 12400 pairs:

$$
p_{N W}(T)=\left(\sum_{i=0}^{N_{s}} k\left(d-O O_{i} \mid T\right)\right) \times\left(\frac{N_{t}-N_{h}}{N_{S}}\right)(3)
$$

Where

1) the $N_{t}$ is the total number of bridge pairs, $N_{h}$ is the high coordination bridge pairs and $N_{s}$ is the sampled bridge pairs. Here, $N_{t}=21057, N_{h}=8657, N_{s}=500$;

2) $k\left(d_{-} O O \mid T\right)$ is the fitted sigmoid function at temperature $T$;

The experiments showed that the J-PtNW performance is 50 times better than $\mathrm{Pt} / \mathrm{C}$ at room temperature at mass activity. We considered that $\mathrm{Pt} / \mathrm{C}$ is $\mathrm{Pt}(111)$ with 6926 surface $\mathrm{Pt}$ atoms, since the J-PtNW has $6926 \mathrm{Pt}$ atoms in total with 3881 on the surface. In this way, we ensure the performance we are comparing is mass activity. This leads to 41,574 pairs. Therefore, the performance of Pt (111) can be estimated as following:

$$
p_{p t(111)}(T)=k\left(d-O O_{0} \mid T\right) \times N_{0}(4)
$$

where $N_{0}=41,574$ is the total number of bridge pairs, and $k$ $\left(d-O O_{0} \mid T\right)$ is the reaction rate for $\mathrm{Pt}(111)$ at temperature.

We then define the improvement of the performance as PI(T), which is dependent of temperature and calculated by formula (5):

$$
P I(T)=p_{N W}(T) / p_{p t(111)}(T)(5)
$$

Figure 5 shows the temperature dependence of the estimated performance improvement. At room temperature, our model leads to PI $=212.21$ for the J-PtNW. This is a factor of 4 higher than the experimental $\mathrm{PI}=50^{29}$. We consider that this agreement validates our explanation. This model, allows us to predict the performance improvement of J-PtNW at increased temperatures. For example, low-temperature PEMFCs use a water-based acidic polymer membrane as the electrolyte, with platinum-based electrodes. Thus the upper temperature limit is around $80{ }^{\circ} \mathrm{C}$. Our model, estimates PI $=19.70$ at $353.15 \mathrm{~K}$ (marked as red diamond in Figure 5). Correcting by the same 
factor 4, we predict the performance of J-PtNW should be around 5 time better than $\mathrm{Pt}(111)$ at $80{ }^{\circ} \mathrm{C}$. This may provide guidance in choosing optimal conditions for the real J-PtNW.

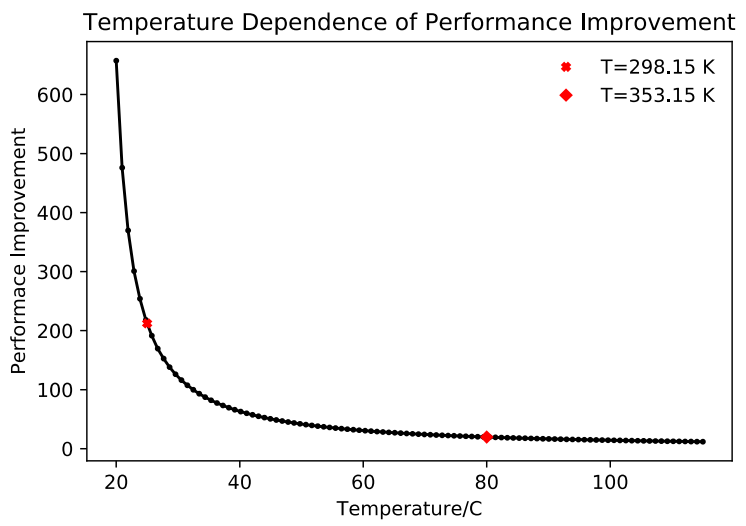

Figure 5. Temperature Dependence of Performance Improvement, which is calculated by formula (4). With increasing temperature, the performance improvement decreases. The PI at $298.15 \mathrm{~K}$ is marked in red, is $\mathrm{PI}=212.21$ is 4 times larger than experimental $=$ 50. The temperature limit of low-temperature PEMFCs is also marked as red diamond, $\mathrm{PI}=19.70$ at $353.15 \mathrm{~K}$.

\subsection{Active Sites Identification}

To reveal the important features of the active sites and to provide insights for designing high-performance electrocatalysts, we characterized the structures of barrier-less sites $(\mathrm{d}-\mathrm{OO}<=2.68 \AA)$. They account for 72 out of the 500 bridge nanoclusters sampled. Among these 72 structures, many share similar geometric features. Thus, we further partitioned them into three subgroups:

I1) Concave-up Rhombus (30.8\%);

I2) Triangle (43.6\%);

I3) Others $(25.6 \%)$.

Figure 6, shows one representative structure from each group. We consider that the Triangle group and Concave-Up Rhombus group play an important role in the dramatically improved performance. We summarized their geometry features and analyzed their statistics among the 500 structures in the following session.

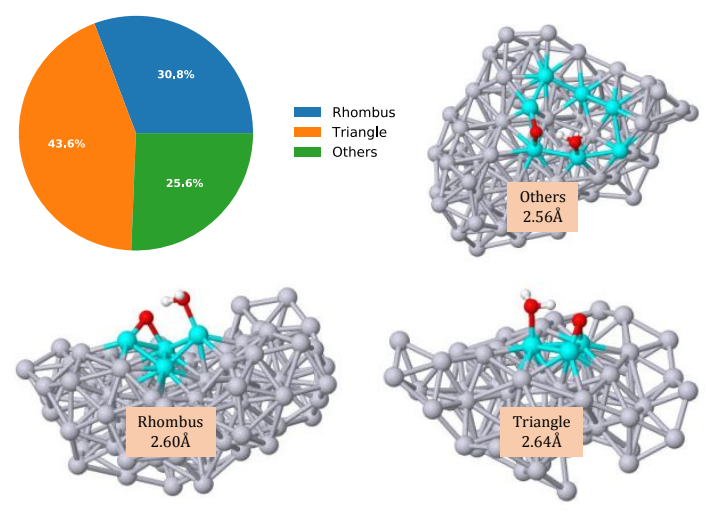

Figure 6. Identification Results of Barrier-Less Sites (d-OO <= $2.68 \AA$ ). Among the 72 barrier-less sites, $30.8 \%$ of them are in the Concave-up Rhombus group and $43.6 \%$ of them are in the Triangle group. One representative structure of each group is attached with their d-OO marked as well.

\subsubsection{Triangle Group Statistics}

As shown in Figure 6, Triangle group I 2 is an important part of barrier-less sites (43.6\%). We define a structure as a triangle structure if its three Pts, the one binding water, and the other two binding O, form a closed ring, marked as cyan in Figure 6. Based on this definition, we picked out all triangle structures from the 500 sampled bridge nanoclusters and plotted their distribution toward d-OO in Figure 7. There are 143 triangles in sample with the main peak located at $\sim 2.75 \AA$. Although several triangles showed large $\mathrm{d}-\mathrm{OO}$ (one representative structure of a bad triangle is shown in Figure 7), we see that major percentage the triangles lead to dramatically improved performance of the J-PtNW. Thus, a synthetic strategy might focus on generating triangle structures. Additional Triangle structures with various d-OO are shown in Figure S11 in Supporting Information.
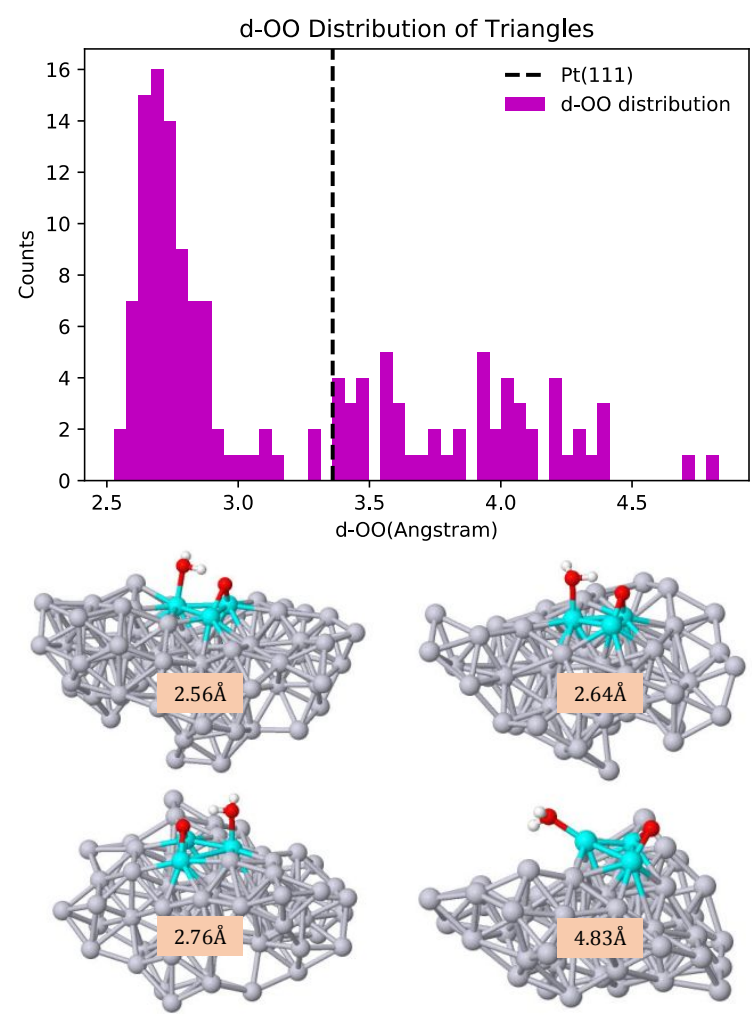

Figure 7. Statistics of Triangle Group among 143 out of 500 Sampled Bridge Nanoclusters. (a) There are 143 Triangles in total, and 87 of them show shorter d-OO than Pt (111). A small fraction of the triangles showed large d-OO, but the main peak is located way left from Pt (111), (dashed line in the plot). (b) Four representative structures of different d-OO. More structures are included in Figure S11 in Supporting Information.

\subsubsection{Concave-Up Rhombus Group Statistics}

We then examined all Concave-up Rhombus structures among the 500 sampled bridge nanoclusters. The Concave-Up Rhombus is defined as following: the three Pts, One Pt binding $\mathrm{H} 2 \mathrm{O}$, and the other two Pts binding $\mathrm{O}$, cannot form a closed ring. Instead another surface $\mathrm{Pt}$ is required to close the ring, forming a concave-up rhombus. As shown in Figure 8, we then define the dihedral angle of a rhombus as the dihedral angle between two surfaces defined by (Pt1, Pt2, Pt4) and (Pt2, Pt3, Pt4). The larger dihedral angle is, the more concave-up the rhombus is. There are a total number of 234 rhombi out of 500 
with different dihedral angles We plotted the correlation between the dihedral angle and d-OO in Figure 8(a), and we

(a)

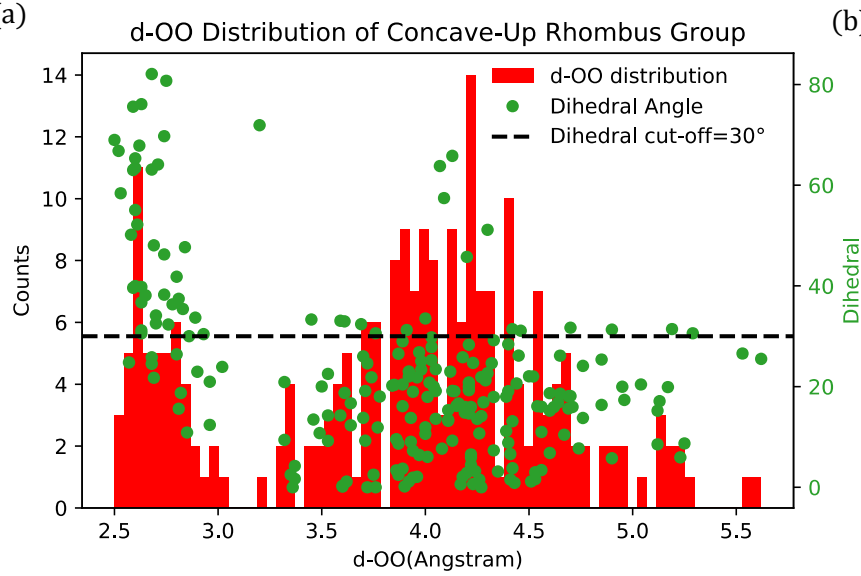

(c)
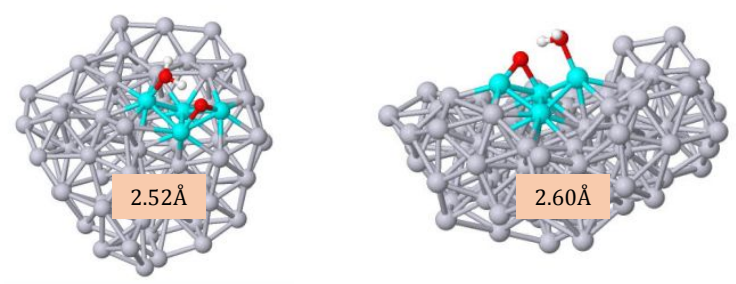

(b)

found the Concave-Up Rhombus with dihedral angle larger than $30^{\circ}$ mostly show short d-OO.

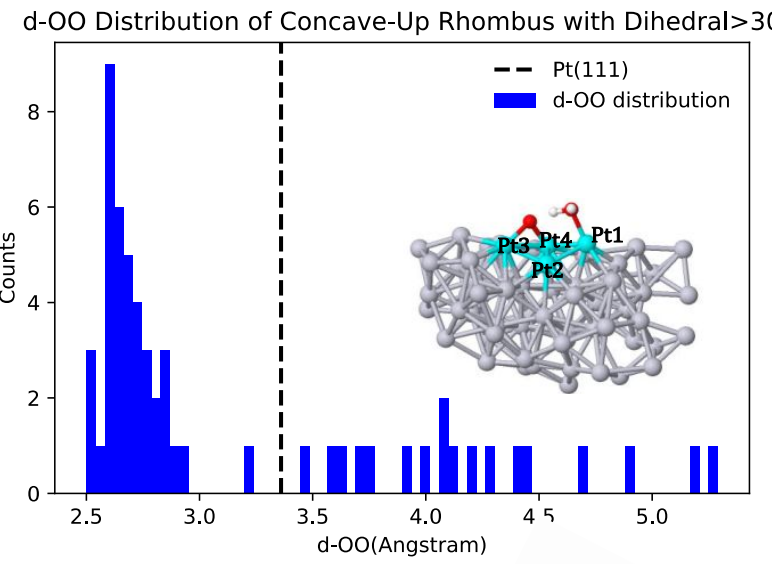

Figure 8. Statistics of Concave-Up Rhombus among 234 out of 500 Sampled Bridge Nanoclusters. (a) The correlation between dihedral angle and d-OO is shown in plot. Here dihedral angle is defined as the angle between two surfaces defined by (Pt1, Pt2, Pt4) and (Pt2, Pt3, $\mathrm{Pt} 4$ ). As we could see, the rhombus with dihedral angle larger than $30^{\circ}$ mostly show short d-OO; (b) Statistics of Concave-up Rhombus with Dihedral Angle $>30^{\circ}$. Most of structures in this groups shows the small d-OO with the main peak located at $2.6 \AA$; (c) Four representative structures with different d-OO are attached here and more structures are available in Figure S12 in Supporting Information.

Thus, if we cut off the dihedral angle to $30^{\circ}$, the population of active Concave-Up Rhombic becomes 57 out of 500 . Therefore, we define our active Concave-Up Rhombic as those with dihedral angle $>30^{\circ}$. These 57 active Concave-Up Rhombi are plotted in the Figure 8(b), along with several representative structures shown in Figure 8(c). More structures with different d-OO distances are available in Figure S12 in Supporting Information

\subsection{Comparison with Previous Literature}

The above discussion, shows the atomistic explanation of the dramatically improved performance. Thus, the jagged NW has many sites with a concave nature that pushes the $\mathrm{OH}$ bond of the $\mathrm{H}_{2} \mathrm{O}_{\text {ads }}$ toward the $\mathrm{O}_{\text {ads }}$, leading to a dramatically reduced energy barrier for $\mathrm{O}_{\text {ads }}$ hydration. Earlier, Huang and coworkers suggested that mechanical strain, high ECSA, and undercoordinated surface Pt atoms may affect this remarkably accelerated ORR activity ${ }^{29}$. Consistent with these previous literature results, our analysis also finds numerous undercoordinated $\mathrm{Pt}$ surface atoms and a high $56.0 \%$ surface atom ratio (Section 2.1), which will affect the ECSA.

To correlate the mechanical strain with catalytic activity, we plotted the $E_{a}$ versus the strain of the selected 18 structures from Section 2.5. Here we used the average Pt-Pt bond length around the adsorption sites to represent the local strain. As shown in Figure S13 of Supporting Information, there is a linear correlation between the catalytic activity and strain. In general, the sites with compressive strains show lower energy barriers and sites with tensile strains show higher energy barriers. The correlation is weak with small $R^{2}$ at 0.46 , but we agree that mechanical strain plays a role in reduced energy barrier of $O_{a d s}$ hydration. Our work, with overall agreement with experiment in NW structure characterization and performance prediction, explains the dramatically improved ORR of J-PtNW from a very different atomistic perspective.

\section{CONCLUSION}

Starting from the J-PtNW synthesized using the ReaxFF reactive force field, we developed the bridge nanocluster model for DFT calculations. Using sites randomly selected from the surface, we observed a strong correlation between d-OO and the free energy barrier $G_{a}$ of the RDS, $O_{a d s}$ hydration. This dramatically reduced the computational cost but accurately described the performance of each bridge pair. We found $14.4 \%$ of the sampled surface sites are barrier-less for the RDS. Identification of these active sites led to two groups sharing similar geometrical patterns, the Triangle group and Concaveup Rhombus. Using the model developed above, we could predict the performance improvement of the whole J-PtNW. We report the atomistic structure of the active sites, which provide some insights in designing high-performance electrocatalysts for ORR.

\section{ASSOCIATED CONTENT}

\section{Supporting Information}

The Supporting Information is available free of charge on the ACS Publications website.

Description of DFT calculations, transition state search used in this work, benchmark calculations for bridge nanocluster model, more 
structures of Triangle and Concave-Up Rhombic Groups and additional calculations. (PDF)

\section{AUTHOR INFORMATION}

\section{Corresponding Author}

*wag@caltech.edu ORCID:0000-0003-0097-5716

\section{Notes}

The authors declare no competing financial interest.

\section{ACKNOWLEDGMENT}

This work was supported by the Joint Center for Artificial Photosynthesis, a DOE Energy Innovation Hub, supported through the Office of Science of the U.S. Department of Energy under Award Number DE-SC0004993. This work uses the Extreme Science and Engineering Discovery Environment(XSEDE), which is supported by National Science Foundation Grant Number ACI1053575, and the computational resources of Caltech High Performance Computing Center (HPC).

\section{REFERENCES}

(1) Peighambardoust, S. J.; Rowshanzamir, S.; Amjadi, M. Review of the Proton Exchange Membranes for Fuel Cell Applications. International Journal of Hydrogen Energy 2010, 35 (17), 93499384. https://doi.org/10.1016/j.ijhydene.2010.05.017.

(2) Steele, B. C. H.; Heinzel, A. Materials for Fuel-Cell Technologies. Nature 2001, 414 (6861), 345-352. https://doi.org/10.1038/35104620.

(3) Wang, S.; Jiang, S. P. Prospects of Fuel Cell Technologies. Natl Sci Rev 2017, 4 (2), 163-166. https://doi.org/10.1093/nsr/nww099.

(4) Shao, M.; Chang, Q.; Dodelet, J.-P.; Chenitz, R. Recent Advances in Electrocatalysts for Oxygen Reduction Reaction. Chem. Rev. 2016, 116 (6), 3594-3657. https://doi.org/10.1021/acs.chemrev.5b00462.

(5) Sui, S.; Wang, X.; Zhou, X.; Su, Y.; Riffat, S.; Liu, C. A Comprehensive Review of Pt Electrocatalysts for the Oxygen Reduction Reaction: Nanostructure, Activity, Mechanism and Carbon Support in PEM Fuel Cells. J. Mater. Chem. A 2017, 5 (5), 1808-1825. https://doi.org/10.1039/C6TA08580F.

(6) He, Q.; Cairns, E. J. Review-Recent Progress in Electrocatalysts for Oxygen Reduction Suitable for Alkaline Anion Exchange Membrane Fuel Cells. J. Electrochem. Soc. 2015, 162 (14), F1504-F1539. https://doi.org/10.1149/2.0551514jes.

(7) Huang, X.; Zhao, Z.; Chen, Y.; Zhu, E.; Li, M.; Duan, X.; Huang, Y. A Rational Design of Carbon-Supported Dispersive Pt-Based Octahedra as Efficient Oxygen Reduction Reaction Catalysts. Energy Environ. Sci. 2014, 7 (9), 2957-2962. https://doi.org/10.1039/C4EE01082E.

(8) Peng, Z.; Yang, H. Designer Platinum Nanoparticles: Control of Shape, Composition in Alloy, Nanostructure and Electrocatalytic Property. Nano Today 2009, 4 (2), 143-164. https://doi.org/10.1016/j.nantod.2008.10.010.

(9) Wu, J.; Yang, H. Platinum-Based Oxygen Reduction Electrocatalysts. Acc. Chem. Res. 2013, 46 (8), 1848-1857. https://doi.org/10.1021/ar300359w.

(10) Porter, N. S.; Wu, H.; Quan, Z.; Fang, J. Shape-Control and Electrocatalytic Activity-Enhancement of Pt-Based Bimetallic Nanocrystals. Acc. Chem. Res. 2013, 46 (8), 1867-1877. https://doi.org/10.1021/ar3002238.

(11) Wang, D.; Xin, H. L.; Hovden, R.; Wang, H.; Yu, Y.; Muller, D. A.; DiSalvo, F. J.; Abruña, H. D. Structurally Ordered Intermetallic Platinum-Cobalt Core-Shell Nanoparticles with Enhanced Activity and Stability as Oxygen Reduction Electrocatalysts. Nat Mater 2013, 12 (1), 81-87. https://doi.org/10.1038/nmat3458.
(12) Mazumder, V.; Chi, M.; More, K. L.; Sun, S. Core/Shell $\mathrm{Pd} / \mathrm{FePt}$ Nanoparticles as an Active and Durable Catalyst for the Oxygen Reduction Reaction. J. Am. Chem. Soc. 2010, 132 (23), 7848-7849. https://doi.org/10.1021/ja1024436.

(13) Stamenkovic, V. R.; Fowler, B.; Mun, B. S.; Wang, G.; Ross, P. N.; Lucas, C. A.; Marković, N. M. Improved Oxygen Reduction Activity on Pt3Ni(111) via Increased Surface Site Availability. Science 2007, 315 (5811), 493-497. https://doi.org/10.1126/science.1135941.

(14) Choi, S.-I.; Xie, S.; Shao, M.; Odell, J. H.; Lu, N.; Peng, H.-C.; Protsailo, L.; Guerrero, S.; Park, J.; Xia, X.; Wang, J.; Kim, M. J.; Xia, Y. Synthesis and Characterization of $9 \mathrm{Nm} \mathrm{Pt-Ni}$ Octahedra with a Record High Activity of $3.3 \mathrm{~A} / \mathrm{MgPt}$ for the Oxygen Reduction Reaction. Nano Lett. 2013, 13 (7), 3420 3425. https://doi.org/10.1021/nl401881z.

(15) Huang, X.; Zhao, Z.; Cao, L.; Chen, Y.; Zhu, E.; Lin, Z.; Li, M.; Yan, A.; Zettl, A.; Wang, Y. M.; Duan, X.; Mueller, T.; Huang, Y. High-Performance Transition Metal-Doped Pt3Ni Octahedra for Oxygen Reduction Reaction. Science 2015, 348 (6240), 1230-1234. https://doi.org/10.1126/science.aaa8765.

(16) Wang, Y.-J.; Zhao, N.; Fang, B.; Li, H.; Bi, X. T.; Wang, H. Carbon-Supported Pt-Based Alloy Electrocatalysts for the Oxygen Reduction Reaction in Polymer Electrolyte Membrane Fuel Cells: Particle Size, Shape, and Composition Manipulation and Their Impact to Activity. Chem. Rev. 2015, 115 (9), 34333467. https://doi.org/10.1021/cr500519c.

(17) Lu, Y.; Thia, L.; Fisher, A.; Jung, C.-Y.; Yi, S. C.; Wang, X. Octahedral PtNi Nanoparticles with Controlled Surface Structure and Composition for Oxygen Reduction Reaction. Sci. China Mater. 2017, 60 (11), 1109-1120. https://doi.org/10.1007/s40843-017-9029-5.

(18) Hasché, F.; Oezaslan, M.; Strasser, P. Activity and Structure of Dealloyed PtNi3 Nanoparticle Electrocatalyst for Oxygen Reduction Reaction in PEMFC. ECS Trans. 2011, 41 (1), 1079 1088. https://doi.org/10.1149/1.3635640.

(19) Mani, P.; Srivastava, R.; Strasser, P. Dealloyed Binary PtM3 $(\mathrm{M}=\mathrm{Cu}, \mathrm{Co}, \mathrm{Ni})$ and Ternary PtNi3M $(\mathrm{M}=\mathrm{Cu}, \mathrm{Co}, \mathrm{Fe}, \mathrm{Cr})$ Electrocatalysts for the Oxygen Reduction Reaction: Performance in Polymer Electrolyte Membrane Fuel Cells. Journal of Power Sources 2011, 196, 666. https://doi.org/10.1016/j.jpowsour.2010.07.047.

(20) Jia, Q.; Caldwell, K.; Strickland, K.; Ziegelbauer, J. M.; Liu, Z.; Yu, Z.; Ramaker, D. E.; Mukerjee, S. Improved Oxygen Reduction Activity and Durability of Dealloyed PtCox Catalysts for Proton Exchange Membrane Fuel Cells: Strain, Ligand, and Particle Size Effects. ACS Catal 2015, 5 (1), 176186. https://doi.org/10.1021/cs501537n.

(21) Strasser, P.; Koh, S.; Yu, C. Voltammetric Surface Dealloying of Pt Bimetallic Nanoparticles: A Novel Synthetic Method Towards More Efficient ORR Electrocatalysts. ECS Trans. 2007, 11 (1), 167-180. https://doi.org/10.1149/1.2780928.

(22) Strasser, P.; Koh, S.; Anniyev, T.; Greeley, J.; More, K.; Yu, C.; Liu, Z.; Kaya, S.; Nordlund, D.; Ogasawara, H.; Toney, M. F.; Nilsson, A. Lattice-Strain Control of the Activity in Dealloyed Core-Shell Fuel Cell Catalysts. Nat Chem 2010, 2 (6), 454-460. https://doi.org/10.1038/nchem.623.

(23) Debe, M. K. Electrocatalyst Approaches and Challenges for Automotive Fuel Cells. Nature 2012, 486 (7401), 43-51. https://doi.org/10.1038/nature11115.

(24) van der Vliet, D.; Wang, C.; Debe, M.; Atanasoski, R.; Markovic, N. M.; Stamenkovic, V. R. Platinum-Alloy Nanostructured Thin Film Catalysts for the Oxygen Reduction Reaction. Electrochimica Acta 2011, 56 (24), 8695-8699. https://doi.org/10.1016/j.electacta.2011.07.063.

(25) Debe, M. K.; Steinbach, A. J.; Vernstrom, G. D.; Hendricks, S. M.; Kurkowski, M. J.; Atanasoski, R. T.; Kadera, P.; Stevens, D. A.; Sanderson, R. J.; Marvel, E.; Dahn, J. R. Extraordinary Oxygen Reduction Activity of Pt3Ni7. J. Electrochem. Soc. 2011, 158 (8), B910-B918. https://doi.org/10.1149/1.3595748. 
(26) Stevens, D. A.; Mehrotra, R.; Sanderson, R. J.; Vernstrom, G. D.; Atanasoski, R. T.; Debe, M. K.; Dahn, J. R. Dissolution of $\mathrm{Ni}$ from High Ni Content Pt1-xNix Alloys. J. Electrochem. Soc. 2011, 158 (8), $\quad$ B905-B909. https://doi.org/10.1149/1.3595747.

(27) van Duin, A. C. T.; Dasgupta, S.; Lorant, F.; Goddard, W. A. ReaxFF: A Reactive Force Field for Hydrocarbons. J. Phys. Chem. A 2001, 105 (41), 9396-9409. https://doi.org/10.1021/jp004368u.

(28) Fortunelli, A.; Goddard III, W. A.; Sementa, L.; Barcaro, G.; Negreiros, F. R.; Jaramillo-Botero, A. The Atomistic Origin of the Extraordinary Oxygen Reduction Activity of Pt3Ni7 Fuel Cell Catalysts. Chemical Science 2015, 6 (7), 3915-3925. https://doi.org/10.1039/C5SC00840A.

(29) Li, M.; Zhao, Z.; Cheng, T.; Fortunelli, A.; Chen, C.-Y.; Yu, R.; Zhang, Q.; Gu, L.; Merinov, B. V.; Lin, Z.; Zhu, E.; Yu, T.; Jia, Q.; Guo, J.; Zhang, L.; Goddard, W. A.; Huang, Y.; Duan, X. Ultrafine Jagged Platinum Nanowires Enable Ultrahigh Mass Activity for the Oxygen Reduction Reaction. Science 2016, 354 (6318), 1414-1419. https://doi.org/10.1126/science.aaf9050.

(30) Cheng, T.; Goddard, W. A.; An, Q.; Xiao, H.; Merinov, B.; Morozov, S. Mechanism and Kinetics of the Electrocatalytic Reaction Responsible for the High Cost of Hydrogen Fuel Cells. Physical Chemistry Chemical Physics 2017, 19 (4), 2666-2673. https://doi.org/10.1039/C6CP08055C.

(31) Noël, J.-M.; Latus, A.; Lagrost, C.; Volanschi, E.; Hapiot, P. Evidence for OH Radical Production during Electrocatalysis of Oxygen Reduction on Pt Surfaces: Consequences and Application. J. Am. Chem. Soc. 2012, 134 (5), 2835-2841. https://doi.org/10.1021/ja211663t.

(32) Anderson, A. B.; Albu, T. V. Catalytic Effect of Platinum on Oxygen Reduction An Ab Initio Model Including Electrode Potential Dependence. J. Electrochem. Soc. 2000, 147 (11), 4229-4238. https://doi.org/10.1149/1.1394046.

(33) Sha, Y.; Yu, T. H.; Merinov, B. V.; Shirvanian, P.; Goddard, W. A. Oxygen Hydration Mechanism for the Oxygen Reduction Reaction at Pt and Pd Fuel Cell Catalysts. J. Phys. Chem. Lett. 2011, 2 (6), 572-576. https://doi.org/10.1021/jz101753e.

(34) Sha, Y.; Yu, T. H.; Merinov, B. V.; Goddard, W. A. Prediction of the Dependence of the Fuel Cell Oxygen Reduction
Reactions on Operating Voltage from DFT Calculations. $J$. Phys. Chem. C 2012, 116 (10), 6166-6173. https://doi.org/10.1021/jp207526u.

(35) Huang, Y.; Chen, Y.; Cheng, T.; Wang, L.-W.; Goddard, W. A. Identification of the Selective Sites for Electrochemical Reduction of $\mathrm{CO}$ to $\mathrm{C}_{2+}$ Products on Copper Nanoparticles by Combining Reactive Force Fields, Density Functional Theory, and Machine Learning. ACS Energy Letters 2018, 2983-2988. https://doi.org/10.1021/acsenergylett.8b01933.

(36) Cheng, T.; Xiao, H.; Goddard, W. A. Nature of the Active Sites for CO Reduction on Copper Nanoparticles; Suggestions for Optimizing Performance. J. Am. Chem. Soc. 2017, 139 (34), 11642-11645. https://doi.org/10.1021/jacs.7b03300.

(37) Chen, Y.; Huang, Y.; Cheng, T.; Goddard, W. A. Identifying Active Sites for CO2 Reduction on Dealloyed Gold Surfaces by Combining Machine Learning with Multiscale Simulations. $J$. Am. Chem. Soc. 2019, 141 (29), 11651-11657. https://doi.org/10.1021/jacs.9b04956.

(38) Cheng, T.; Huang, Y.; Xiao, H.; Goddard, W. A. Predicted Structures of the Active Sites Responsible for the Improved Reduction of Carbon Dioxide by Gold Nanoparticles. The Journal of Physical Chemistry Letters 2017, 8 (14), 3317-3320. https://doi.org/10.1021/acs.jpclett.7b01335.

(39) Kresse, G.; Hafner, J. Ab Initio Molecular-Dynamics Simulation of the Liquid-Metal--Amorphous-Semiconductor Transition in Germanium. Phys. Rev. B 1994, 49 (20), 1425114269. https://doi.org/10.1103/PhysRevB.49.14251.

(40) Henkelman, G.; Uberuaga, B. P.; Jónsson, H. A Climbing Image Nudged Elastic Band Method for Finding Saddle Points and Minimum Energy Paths. J. Chem. Phys. 2000, 113 (22), 9901-9904. https://doi.org/10.1063/1.1329672.

(41) Mathew, K.; Sundararaman, R.; Letchworth-Weaver, K.; Arias, T. A.; Hennig, R. G. Implicit Solvation Model for DensityFunctional Study of Nanocrystal Surfaces and Reaction Pathways. J. Chem. Phys. 2014, 140 (8), 084106. https://doi.org/10.1063/1.4865107. 


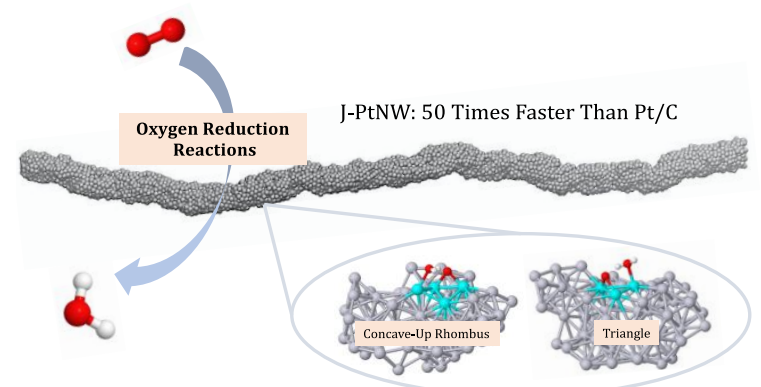

